Conflict of interest: None declared.

Acknowledgements: The authors thank the National Health Research Institute of Taiwan for providing the NHIRD. Details of the computer code for statistical analyses are available from the corresponding author.

\title{
References
}

1 Wang JY, Lee LN, Lai HC, et al. Fluoroquinolone resistance in Mycobacterium tuberculosis isolates: associated genetic mutations and relationship to antimicrobial exposure. J Antimicrob Chemother 2007; 59: 860-865.

2 Wang JY, Hsueh PR, Jan IS, et al. Empirical treatment with a fluoroquinolone delays the treatment for tuberculosis and is associated with a poor prognosis in endemic areas. Thorax 2006; 61: 903-908.

3 Migliori GB, Zellweger JP, Abubakar I, et al. European union standards for tuberculosis care. Eur Respir J 2012; 39: 807-819.

TB CARE I. International Standards for Tuberculosis Care (ISTC). 3rd Edn. The Hague, TB CARE I, 2014.

5 Migliori GB, Langendam MW, D’Ambrosio L, et al. Protecting the tuberculosis drug pipeline: stating the case for the rational use of fluoroquinolones. Eur Respir J 2012; 40: 814-822.

6 Migliori GB, Lienhardt C, Weyer K, et al. Ensuring rational introduction and responsible use of new TB tools: outcome of an ERS multisector consultation. Eur Respir J 2014; 44: 1412-1417.

7 Tseng YT, Chuang YC, Shu CC, et al. Empirical use of fluoroquinolones improves the survival of critically ill patients with tuberculosis mimicking severe pneumonia. Crit Care 2012; 16: R207.

8 National Health Insurance Administration, Ministry of Health and Welfare. The National Health Insurance Statistics, 2013. www.nhi.gov.tw/English/webdata/webdata.aspx?menu=11\&menu_id=296\&webdata_id=1942\&WD_ID=296 Date last updated: March 25, 2015.

9 Lee $\mathrm{CH}$, Lee MC, Shu CC, et al. Risk factors for pulmonary tuberculosis in patients with chronic obstructive airway disease in Taiwan: a nationwide cohort study. BMC Infect Dis 2013; 13: 194.

10 Wang JY, Lee MC, Shu CC, et al. Optimal duration of anti-tuberculosis treatment in diabetic patients: nine or six months? Chest 2015; 147: 520-528.

11 Migliori GB, Sotgiu G, Blasi F, et al. Towards the development of EU/EEA Standards for Tuberculosis Care (ESTC). Eur Respir J 2011; 38: 493-495.

12 Frei CR, Labreche MJ, Attridge RT. Fluoroquinolones in community-acquired pneumonia: guide to selection and appropriate use. Drugs 2011; 71: 757-770.

13 Centers for Disease Control, Department of Health, R.O.C. (Taiwan). Taiwan Tuberculosis Control Report 2013. www.cdc.gov.tw/uploads/files/201407/103228a0-fadd-47b0-b056-8dedda9fceld.pdf Date last updated: April 2014.

14 Nyamande K, Lalloo UG, John M. TB presenting as community-acquired pneumonia in a setting of high TB incidence and high HIV prevalence. Int J Tuberc Lung Dis 2007; 11: 1308-1313.

15 Copeland KT, Checkoway H, McMichael AJ, et al. Bias due to misclassification in the estimation of relative risk. Am J Epidemiol 1977; 105: 488-495.

\section{Antipyretic effect of dexamethasone in community-acquired pneumonia}

To the Editor:

The cornerstones of treatment for community-acquired pneumonia (CAP) are early diagnosis and initiation of appropriate antibiotic therapy [1]. Despite prevention with vaccination, and optimal antibiotic treatment, CAP is associated with high mortality and morbidity and significant healthcare costs $[2,3]$. Adjunctive therapy for CAP could help to reduce disease severity and, indeed, the addition of dexamethasone to antibiotic treatment in patients hospitalised with CAP has shown to reduce the length of hospital stay by 1 day [4]. Recent trials showed similar results [5, 6]. One of the comments raised to our previous study was that the antipyretic effect of dexamethasone might be the major underlying explanation for this result [7]. In patients, hospitalised with CAP, body temperature measurement is part of the standard care. Stable defervescence is one of the criteria used to define clinical stability [8]. Other clinical markers used in the decision to discharge a patient are respiratory and haemodynamic stability, the ability to maintain oral intake and a normal mental status [9-11]. Besides white cell count, C-reactive protein can be a useful marker of treatment response $[10,11]$.

In a post hoc analysis, we assessed the effect of dexamethasone on body temperature in our previously conducted trial [4] and studied whether its antipyretic properties may have influenced the length of stay in hospital. 
In the original clinical trial, patients, hospitalised with CAP, were randomised to a 4-day course of $5 \mathrm{mg}$ intravenous dexamethasone or placebo, both in addition to standard care. More details of the study protocol are described elsewhere [4]. Retrospectively, we collected body temperature registrations from the medical charts of the study participants. Our aim was to collect three temperature measurements during each day of admission per patient. Differences in body temperature were assessed using Students' t-test. We analysed the time to persistent defervescence, defined as a body temperature $<37.8^{\circ} \mathrm{C}$ for $8 \mathrm{~h}$ consecutively, with the chi-square test and with the Kaplan-Meier method and a log-rank test.

Body temperature registrations could be retrieved from 143 patients (94.7\%) in the dexamethasone group and 137 patients $(89.5 \%)$ in the placebo group. Baseline patient characteristics are evenly distributed over both treatment arms and reflect the characteristics in the original trial. The amount of patients that received antibiotic therapy prior to hospital admission did not differ between groups. Neither did the duration.

In the dexamethasone group, body temperature already started to decrease on the day of admission, whereas the decrease in the placebo group commenced 1 day later (fig. 1). At the end of the admission day the mean $\pm \mathrm{SD}$ for temperature was $37.68 \pm 1.04^{\circ} \mathrm{C}$ in the dexamethasone group and $38.0 \pm 1.072^{\circ} \mathrm{C}$ in the placebo group (95\% CI $0.07-0.61, \mathrm{p}=0.015$ ). The mean time to persistent defervescence was significantly shorter in patients treated with dexamethasone ( 0.97 days versus 1.77 days, $\mathrm{p}<0.001)$. The temperature difference between the dexamethasone and placebo group persisted until the end of day 4 of hospitalisation ( $24 \mathrm{~h}$ after the last administration of the study medication). Average body temperature from day 1 to day 4, during hospitalisation, was $37.3^{\circ} \mathrm{C}$ and $36.9^{\circ} \mathrm{C}$ in the placebo group and dexamethasone group, respectively. On every day from day 0 until day 4 , the cumulative proportion of patients that reached defervescence was significantly higher in the dexamethasone group. By day 4, all patients in the dexamethasone group had reached stable defervescence, while $10.3 \%$ in the placebo group still had a fever $(\mathrm{p}<0.05)$ (table 1$)$. The number of patients discharged did not diverge during the period that dexamethasone and placebo were given (fig. 1). Discharge rates did not start diverging until day 7 and day $8(\mathrm{p}<0.05)$ (table 1$)$.

We found that body temperature was significantly suppressed in patients treated with dexamethasone. This resulted in a shorter time to persistent defervescence, one of the criteria used to define clinical stability [10]. There was no difference in discharge rates between the study groups during the period that a temperature difference was present. The difference in discharge rates between study groups started to occur from day 5 onwards. At that time, body temperature no longer differed between the two study groups. The most likely mechanism by which body temperature is suppressed in patients treated with dexamethasone is the suppression of pro-inflammatory and pyretic cytokines $[12,13]$. The courses of C-reactive protein, interleukin (IL)-6 and IL-10 from patients included in this study have been shown

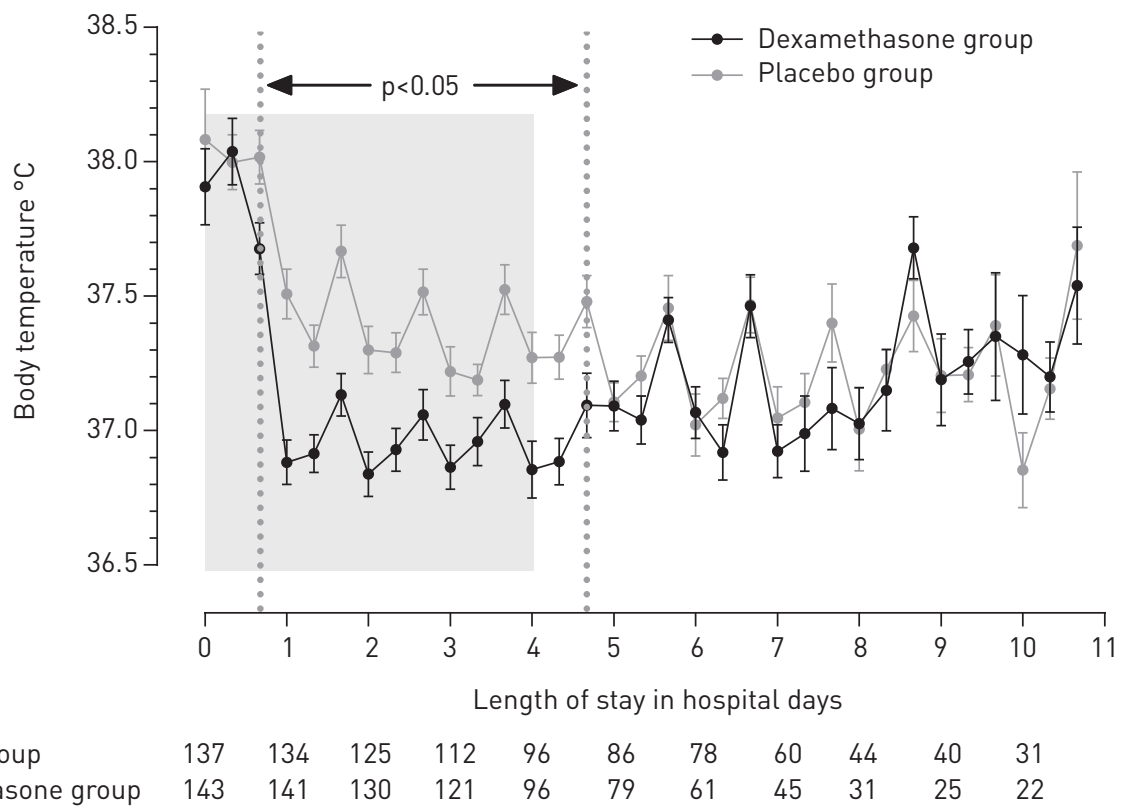

FIGURE 1 Mean morning, afternoon and evening body temperatures from hospital admission to day 10. Dotted lines show the period during which differences in body temperature between groups are significant $(\mathrm{p}<0.05)$. Grey box indicates the period of dexamethasone or placebo administration. Error bars show standard error. 
TABLE 1 Cumulative percentages of patients free of fever per day of admission ${ }^{\#}$ and of patients discharged on each day of admission

\begin{tabular}{|c|c|c|c|c|}
\hline \multirow[t]{2}{*}{ Day } & \multicolumn{2}{|c|}{ Dexamethasone $n=143$} & \multicolumn{2}{|c|}{ Placebo $n=137$} \\
\hline & Free of fever & Patients discharged & Free of fever & Patients discharged \\
\hline 0 & 15.9 & 0 & $6.4^{*}$ & 0 \\
\hline 1 & 89.8 & 0 & $67.9 *$ & 0.7 \\
\hline 2 & 98.9 & 0.7 & $79.5^{*}$ & 1.5 \\
\hline 3 & 98.9 & 4.2 & $88.5^{*}$ & 4.4 \\
\hline 4 & 100 & 12.6 & $89.7^{*}$ & 13.1 \\
\hline 5 & 100 & 25.9 & $96.2^{9}$ & 23.4 \\
\hline 6 & 100 & 42 & 97.4 & $32.1^{\pi}$ \\
\hline 7 & 100 & 56.6 & 97.4 & $42.3^{*}$ \\
\hline 8 & 100 & 64.3 & 100 & $52.6^{*}$ \\
\hline 9 & 100 & 74.8 & 100 & 67.2 \\
\hline 10 & 100 & 76.9 & 100 & 70.1 \\
\hline
\end{tabular}

\#: based on two consecutive readings. *: $p<0.05 ;{ }^{n}: p<0.1$, dexamethasone versus placebo.

elsewhere [4]. In accordance with our findings, SNIJDERs et al. [14] found that defervescence (and C-reactive protein decrease) was reached faster in hospitalised patients with CAP, treated with $40 \mathrm{mg}$ of prednisolone for a total of 7 days, when compared to placebo-treated hospitalised patients (median $\pm \mathrm{SD}$ $2 \pm 1$ days versus $3 \pm 2$ days, $\mathrm{p}<0.01$ ).

In a comment on our trial it was suggested that the "defervescence caused by the corticosteroids" might be the explanation for the reduced length of hospital stay in patients who received dexamethasone $[4,7]$. The fact that discharge rates started to diverge at day 7 and that by that time temperature differences had disappeared for days (table 1), makes such an explanation unlikely. However, a delayed effect on discharge decision making cannot be ruled out. Absence of hypothermia or hyperthermia was only one of the criteria used to define clinical stability in the original trial. The other criteria that were used as a rule for hospital discharge were: improvement of shortness of breath, a consistent decrease of C-reactive protein concentrations, and adequate oral intake and gastrointestinal absorption [4]. Except for C-reactive protein concentration, daily registration of these criteria was not part of the original study protocol. It was thereby commented upon that the daily measurement of C-reactive protein may have influenced the length of stay in hospital [15]. Together with concurrent body temperature suppression, this might have favoured dexamethasone.

The main limitation of our study is that the body temperature measurements, taken during the hospital stay, were not part of the original study protocol. Consequently, body temperature values could not be retrieved for all patients at every time point. However, the occurrence of missing data can be considered fully non-differential because of the randomised nature of the trial. A point of critique to our concept of determining defervescence might be that temperature can increase again after two consecutive measures $<37.8^{\circ} \mathrm{C}$. Only two patients in the dexamethasone group and 11 patients in the placebo group developed fever after being labelled as having stable defervescence. Furthermore, the prescription of paracetamol, often prescribed because of its antipyretic effect, was not registered. We can, therefore, only speculate on the paracetamol effect. If any difference did occur, the prescription rate of paracetamol might be expected to be higher in the placebo group, since defervescence was more rapidly attained in patients treated with dexamethasone. It is highly unlikely that the unevenly distributed use of paracetamol would account for the faster defervescence in the dexamethasone treated patients.

In summary, our findings support the hypothesis that the reduction in length of stay in hospital was the result of a faster, overall, clinical recovery from pneumonia caused by dexamethasone, rather than a "cosmetic" suppression of fever.

(7) @ERSpublications

Antipyretic effect of dexamethasone in community-acquired pneumonia does not explain reduced length of hospital stay http://ow.ly/LTcST

Stefan M.T. Vestjens ${ }^{1}$, Simone M.C. Spoorenberg ${ }^{1}$, Ger T. Rijkers ${ }^{2,3}$, Jan C. Grutters ${ }^{4}$, Ewoudt M.W. van de Garde ${ }^{5,6}$, Sabine C.A. Meijvis ${ }^{1}$ and Willem Jan W. Bos ${ }^{1}$

${ }^{1}$ Dept of Internal Medicine, St. Antonius Hospital, Nieuwegein, The Netherlands. ${ }^{2}$ Dept of Medical Microbiology and Immunology, St. Antonius Hospital, Nieuwegein, The Netherlands. ${ }^{3}$ Science Dept, University College Roosevelt, 
Middelburg, The Netherlands. ${ }^{4}$ Dept of Pulmonology, St. Antonius Hospital, Nieuwegein, The Netherlands. ${ }^{5}$ Division of Pharmacoepidemiology and Clinical Pharmacology, University of Utrecht, Utrecht, The Netherlands. ${ }^{6}$ Dept of Clinical Pharmacy, St Antonius Hospital, Nieuwegein, The Netherlands.

Correspondence: Stefan M.T. Vestjens, Dept of Internal Medicine, St. Antonius Hospital, Koekoekslaan 1, 3430 EM Nieuwegein, P.O. Box 2500, The Netherlands. E-mail: s.vestjens@antoniusziekenhuis.nl

Received: Dec 072014 | Accepted after revision: March 28 2015 | First published online: May 282015

Conflict of interest: None declared.

Acknowledgements: We acknowledge the substantial contribution of Douwe H. Biesma (Dept of Internal Medicine St. Antonius Hospital, Nieuwegein and Dept of Internal Medicine, University Medical Centre Utrecht, Utrecht, The Netherlands), Hans Hardeman (Dept of Pulmonology, St. Antonius Hospital, Nieuwegein, The Netherlands), Rik Heijligenberg (Dept of Internal Medicine, Gelderse Vallei Hospital, Ede, The Netherlands), Hilde H.F. Remmelts (Dept of Internal Medicine, University Medical Centre Utrecht, Utrecht and Dept of Internal Medicine, Gelderse Vallei Hospital, Ede, The Netherlands), Heleen van Velzen-Blad and G. Paul Voorn (both Dept of Medical Microbiology and Immunology, St. Antonius Hospital, Nieuwegein, The Netherlands), to the original trial.

\section{References}

1 Garnacho-Montero J, Garcia-Cabrera E, Diaz-Martin A, et al. Determinants of outcome in patients with bacteraemic pneumococcal pneumonia: importance of early adequate treatment. Scand J Infect Dis 2010; 42: 185-192.

2 Gil-Prieto R, Garcia-Garcia L, Alvaro-Meca A, et al. The burden of hospitalisations for community-acquired pneumonia (CAP) and pneumococcal pneumonia in adults in Spain (2003-2007). Vaccine 2011; 29: 412-416.

3 Spoorenberg SM, Bos WJ, Heijligenberg R, et al. Microbial aetiology, outcomes, and costs of hospitalisation for community-acquired pneumonia; an observational analysis. BMC Infect Dis 2014; 14: 335.

4 Meijvis SC, Hardeman $\mathrm{H}$, Remmelts $\mathrm{HH}$, et al. Dexamethasone and length of hospital stay in patients with community-acquired pneumonia: a randomised, double-blind, placebo-controlled trial. Lancet 2011; 377: 2023-2030.

5 Blum CA, Nigro N, Briel M, et al. Adjunct prednisone therapy for patients with community-acquired pneumonia: a multicentre, double-blind, randomised, placebo-controlled trial. Lancet 2015; 385: 1511-1518.

6 Torres A, Sibila O, Ferrer M, et al. Effect of corticosteroids on treatment failure among hospitalized patients with severe community-acquired pneumonia and high inflammatory response: a randomized clinical trial. JAMA 2015; 313: 677-686.

7 Joseph L, Goldberg S, Picard E. Dexamethasone in community-acquired pneumonia. Lancet 2011; 378: 980

8 Halm EA, Fine MJ, Kapoor WN, et al. Instability on hospital discharge and the risk of adverse outcomes in patients with pneumonia. Arch Intern Med 2002; 162: 1278-1284.

9 Wiersinga WJ, Bonten MJ, Boersma WG, et al. SWAB/NVALT (Dutch Working Party on Antibiotic Policy and Dutch Association of Chest Physicians) guidelines on the management of community-acquired pneumonia in adults. Neth J Med 2012; 70: 90-101.

10 Woodhead M, Blasi F, Ewig S, et al. Guidelines for the management of adult lower respiratory tract infections full version. Clin Microbiol Infect 2011; 17: Suppl. 6, E1-E59.

11 Lim WS, Baudouin SV, George RC, et al. BTS guidelines for the management of community acquired pneumonia in adults: update 2009. Thorax 2009; 64: Suppl. 3, iiil-iii55.

12 Endeman H, Meijvis SC, Rijkers GT, et al. Systemic cytokine response in patients with community-acquired pneumonia. Eur Respir J 2011; 37: 1431-1438.

13 Remmelts HH, Meijvis SC, Biesma DH, et al. Dexamethasone downregulates the systemic cytokine response in patients with community-acquired pneumonia. Clin Vaccine Immunol 2012; 19: 1532-1538.

14 Snijders D, Daniels JM, de Graaff CS, et al. Efficacy of corticosteroids in community-acquired pneumonia: a randomized double-blinded clinical trial. Am J Respir Crit Care Med 2010; 181: 975-982.

15 Meybeck A, Turbelin C, Delannoy PY, et al. Dexamethasone in community-acquired pneumonia. Lancet 2011; 378: 980 . 